\title{
AN EXTRAPOLATION METHOD FOR A CLASS OF BOUNDARY INTEGRAL EQUATIONS
}

\author{
YUESHENG XU AND YUNHE ZHAO
}

\begin{abstract}
Boundary value problems of the third kind are converted into boundary integral equations of the second kind with periodic logarithmic kernels by using Green's formulas. For solving the induced boundary integral equations, a Nyström scheme and its extrapolation method are derived for periodic Fredholm integral equations of the second kind with logarithmic singularity. Asymptotic expansions for the approximate solutions obtained by the Nyström scheme are developed to analyze the extrapolation method. Some computational aspects of the methods are considered, and two numerical examples are given to illustrate the acceleration of convergence.
\end{abstract}

\section{INTRODUCTION AND PRELIMINARIES}

In this paper, we establish an extrapolation method for the boundary integral equation induced from the boundary value problem of the third kind:

$$
\begin{gathered}
\triangle u(P)=0, \quad P \in D, \\
\frac{\partial u(P)}{\partial \mathbf{n}_{P}}=-c u(P)+g(P), \quad P \in \Gamma:=\partial D,
\end{gathered}
$$

where $D$ is a bounded, simply connected open region in $R^{2}$ with a smooth boundary $\Gamma$. We seek a solution $u \in C^{2}(D) \cap C^{1}(\bar{D})$ for the boundary value problem (1.1)(1.2). In (1.2), $\mathbf{n}_{P}$ denotes the exterior unit normal to $\Gamma$ at $P$, the function $g$ is assumed given and continuous on $\Gamma$, and $c$ is a positive constant. This is the linear version of the boundary value problem considered in [5]. A survey [4] of boundary integral equation methods in $R^{3}$ will help the reader to get an insight into the connection between boundary value problems and the corresponding integral equations.

Using Green's representation formula for harmonic functions, we show as in [5] that the function $u$ satisfies

$$
u(P)=\frac{1}{2 \pi} \int_{\Gamma} u(Q) \frac{\partial}{\partial \mathbf{n}_{Q}}[\log |P-Q|] d \sigma(Q)-\frac{1}{2 \pi} \int_{\Gamma} \frac{\partial u(Q)}{\partial \mathbf{n}_{Q}} \log |P-Q| d \sigma(Q)
$$

Received by the editor February 21, 1994 and, in revised form, October 4, 1994.

1991 Mathematics Subject Classification. Primary 65R20, 65B05, 45L10.

Key words and phrases. Boundary value problem, boundary integral equations, EulerMaclaurin formula, extrapolation scheme, Nyström method, periodic logarithmic Fredholm integral equations, asymptotic expansion.

This work is partially supported by NASA under grant NAG 3-1312. 
for all $P \in D$, where $d \sigma(Q)$ denotes the differential of the line element along $\Gamma$ with respect to the point $Q$. Letting $P$ tend to a point on $\Gamma$, and using the boundary condition in (1.2), we obtain

$$
\begin{aligned}
u(P) & -\frac{1}{\pi} \int_{\Gamma} u(Q) \frac{\partial}{\partial \mathbf{n}_{Q}}[\log |P-Q|] d \sigma(Q)-\frac{c}{\pi} \int_{\Gamma} u(Q) \log |P-Q| d \sigma(Q) \\
& =-\frac{1}{\pi} \int_{\Gamma} g(Q) \log |P-Q| d \sigma(Q), \quad P \in \Gamma
\end{aligned}
$$

Then we can solve the boundary integral equation (1.4) for $u$ on $\Gamma$ and obtain the normal derivative from (1.2). Finally, the representation (1.3) gives $u(P)$ for $P \in D$. It is required for the use of (1.3)-(1.4) that the transfinite diameter of $\Gamma$, denoted by $C_{\Gamma}$, not be equal to 1 . If it is 1 , then (1.1)-(1.2) can be redefined on a rescaled region $D$ in such a way that the new $C_{\Gamma} \neq 1$ (see [5]). The solvability of (1.4) follows from the results of [10].

With the operator notation

$$
(A v)(P)=\frac{1}{\pi} \int_{\Gamma} v(Q) \frac{\partial}{\partial n_{Q}} \log |P-Q| d \sigma(Q), P \in \Gamma,
$$

and

$$
(B v)(P)=\frac{c}{\pi} \int_{\Gamma} v(Q) \log |P-Q| d \sigma(Q), P \in \Gamma,
$$

equation (1.4) is written symbolically as

$$
u(P)-(A u)(P)-(B u)(P)=-\frac{1}{c}(B g)(P), \quad P \in \Gamma .
$$

We introduce a parametrization

$$
\mathbf{r}(t)=(\xi(t), \eta(t)), \quad 0 \leq t \leq 2 \pi,
$$

for the boundary $\Gamma$. Assume that each component of $\mathbf{r}$ is in $C_{2 \pi}^{\infty}(-\infty, \infty)$, the space of $2 \pi$-periodic functions in $C^{\infty}$, with $\left|\mathbf{r}^{\prime}(t)\right|=\sqrt{\xi^{\prime}(t)^{2}+\eta^{\prime}(t)^{2}} \neq 0$ for $0 \leq t \leq 2 \pi$. Using this parametrization, we rewrite the operators $A$ and $B$ as

$$
(A v)(t)=\frac{1}{\pi} \int_{0}^{2 \pi} \frac{\eta^{\prime}(s)[\xi(s)-\xi(t)]-\xi^{\prime}(s)[\eta(s)-\eta(t)]}{[\xi(s)-\xi(t)]^{2}+[\eta(s)-\eta(t)]^{2}} v(s) d s
$$

and

$$
\begin{aligned}
(B v)(t)= & \frac{c}{\pi} \int_{0}^{2 \pi} v(s)\left|\mathbf{r}^{\prime}(s)\right| \log |\mathbf{r}(t)-\mathbf{r}(s)| d s \\
= & \frac{c}{\pi} \int_{0}^{2 \pi} v(s)\left|\mathbf{r}^{\prime}(s)\right|\{\log |t-s|+\log |2 \pi-s+t|+\log |2 \pi-t+s|\} d s \\
& +\frac{c}{\pi} \int_{0}^{2 \pi} v(s)\left|\mathbf{r}^{\prime}(s)\right| \log \left[\frac{|\mathbf{r}(t)-\mathbf{r}(s)|}{|t-s||2 \pi-s+t||2 \pi-t+s|}\right] d s
\end{aligned}
$$

for $v \in C_{2 \pi}[0,2 \pi]$, where $C_{2 \pi}[0,2 \pi]$ denotes the subspace of $2 \pi$-periodic functions in $C[0,2 \pi]$. We denote by $a(t, s)$ the kernel of the operator $A$. When $s=t+2 l \pi$ with an integer $l$, then

$$
a(t, t+2 l \pi)=\frac{\xi^{\prime}(t) \eta^{\prime \prime}(t)-\eta^{\prime}(t) \xi^{\prime \prime}(t)}{2 \pi\left[\xi^{\prime}(t)^{2}+\eta^{\prime}(t)^{2}\right]}
$$


Moreover, $a(t, s)$ is in $C_{2 \pi}^{\infty}(-\infty, \infty)$. In fact, it is clear that for $s \neq t+2 l \pi, a(t, s)$ is infinitely many times differentiable. To see that it is also differentiable at $s=$ $t+2 l \pi$, we consider both numerator and denominator of $a(t, s)$ as functions of $s$ and represent them by their Taylor expansions at $s=t+2 l \pi$. Then we find

$$
a(t, s)=\frac{\frac{1}{2}\left(\eta^{\prime \prime}(t) \xi^{\prime}(t)-\xi^{\prime \prime}(t) \eta^{\prime}(t)\right)+O(s-(t+2 l \pi))}{\xi^{\prime}(t)^{2}+\eta^{\prime}(t)^{2}+O(s-(t+2 l \pi))} .
$$

Since the denominator of the right-hand side converges to $\xi^{\prime}(t)^{2}+\eta^{\prime}(t)^{2} \neq 0$ as $s \rightarrow t+2 l \pi$, one can see that for any integer $n \geq 0$ the $n$th derivative of the righthand side of the above equation at $s=t+2 l \pi$ exists. Since $\xi$ and $\eta$ are $2 \pi$-periodic, $a(t, s)$ is $2 \pi$-periodic. Hence, we conclude that $a(t, s)$ is in $C_{2 \pi}^{\infty}(-\infty, \infty)$. In $(1.9)$, $\log |t-s|$ has a singularity along the diagonal, $\log |2 \pi-s+t|$ and $\log |2 \pi-t+s|$ have singularities at $s=t+2 \pi$ and $s=t-2 \pi$, respectively. Let

$$
b(t, s):=\log \left[\frac{|\mathbf{r}(t)-\mathbf{r}(s)|}{|t-s||2 \pi-s+t||2 \pi-t+s|}\right] .
$$

Then it can be proved that

$$
b(t, s) \in \hat{C}^{\infty}:=C^{\infty}(\{(t, s):|t-s| \leq 3 \pi, t \in(-\infty, \infty)\}) .
$$

In fact, for $s \neq t, t+2 \pi, t-2 \pi, b(t, s)$ is infinitely many times differentiable. To see that it is also infinitely many times differentiable at $s=t$, we consider $|\mathbf{r}(t)-\mathbf{r}(s)|^{2}$ as a function of $s$ and represent it by its Taylor expansion at $s=t$; we find

$$
b(t, s)=\frac{1}{2} \log \left[\frac{\xi^{\prime}(t)^{2}+\eta^{\prime}(t)^{2}+O(s-t)}{(2 \pi-s+t)^{2}(2 \pi-t+s)^{2}}\right] .
$$

Clearly, the right-hand side of the above equation is infinitely many times differentiable at $s=t$. Similarly, one can see that $b(t, s)$ is also infinitely many times differentiable at $s=t+2 \pi$ and $s=t-2 \pi$.

Let $K=A+B$. For $v \in C_{2 \pi}[0,2 \pi]$, we have

$$
(K v)(t)=\int_{0}^{2 \pi} k(t, s) v(s) d s
$$

where

$$
\begin{aligned}
k(t, s)= & \frac{c}{\pi}\left|\mathbf{r}^{\prime}(s)\right| \log |t-s|+\frac{c}{\pi}\left|\mathbf{r}^{\prime}(s)\right| \log |2 \pi-s+t|+\frac{c}{\pi}\left|\mathbf{r}^{\prime}(s)\right| \log |2 \pi-t+s| \\
+ & \left\{\frac{c}{\pi}\left|\mathbf{r}^{\prime}(s)\right| \log \left[\frac{|\mathbf{r}(t)-\mathbf{r}(s)|}{|t-s||2 \pi-s+t||2 \pi-t+s|}\right]\right. \\
& \left.+\frac{1}{\pi} \frac{\eta^{\prime}(s)[\xi(s)-\xi(t)]-\xi^{\prime}(s)[\eta(s)-\eta(t)]}{[\xi(s)-\xi(t)]^{2}+[\eta(s)-\eta(t)]^{2}}\right\}, \quad t, s \in[0,2 \pi] .
\end{aligned}
$$

In operator notation, equation (1.7) becomes

$$
u(\mathbf{r}(t))-(K(u \circ \mathbf{r}))(t)=-\frac{1}{c}(B(g \circ \mathbf{r}))(t), \quad t \in[0,2 \pi] .
$$


The following lemma gives the differentiability of the function on the right-hand side of (1.10). We denote by $C_{2 \pi}^{n}(-\infty, \infty)$ the subspace of $2 \pi$-periodic functions in $C^{n}(-\infty, \infty)$.

Lemma 1.1. If $(g \circ \mathbf{r}) \in C_{2 \pi}^{n}(-\infty, \infty)$, then $B(g \circ \mathbf{r}) \in C_{2 \pi}^{n}(-\infty, \infty)$.

Proof. Let $F(s)=g(\mathbf{r}(s))\left|\mathbf{r}^{\prime}(s)\right|$. Applying the operator $B$ to $(g \circ \mathbf{r})$ yields

$$
(B(g \circ \mathbf{r}))(t)=\frac{c}{\pi} \int_{0}^{2 \pi} F(s) \log |\mathbf{r}(t)-\mathbf{r}(s)| d s .
$$

Since $F(s) \log |\mathbf{r}(t)-\mathbf{r}(s)|$ is periodic in both $t$ and $s$ with period $2 \pi, B(g \circ \mathbf{r})$ is periodic with period $2 \pi$. We shall complete our proof by showing that for any $\alpha \in R$, we have $B(g \circ \mathbf{r}) \in C^{n}(\alpha, 2 \pi+\alpha)$, which evidently implies the conclusion of the lemma. Noticing the periodicity of $F(s) \log |\mathbf{r}(t)-\mathbf{r}(s)|$, we have

$$
(B(g \circ \mathbf{r}))(t)=\frac{c}{\pi} \int_{\alpha}^{2 \pi+\alpha} F(s) \log |\mathbf{r}(t)-\mathbf{r}(s)| d s, \quad t \in(\alpha, 2 \pi+\alpha),
$$

that is,

$$
\begin{aligned}
&(B(g \circ \mathbf{r}))(t)= \frac{c}{\pi} \int_{\alpha}^{2 \pi+\alpha} F(s)\{\log |t-s|+\log |2 \pi-s+t|+\log |2 \pi-t+s|\} d s \\
&+\frac{c}{\pi} \int_{\alpha}^{2 \pi+\alpha} F(s) \log \left[\frac{|\mathbf{r}(t)-\mathbf{r}(s)|}{|t-s||2 \pi-s+t||2 \pi-t+s|}\right] d s, \\
& t \in(\alpha, 2 \pi+\alpha) .
\end{aligned}
$$

It has been proved that the kernel $b(t, s)$ of the last integral in the right-hand side of the equation above is in $\hat{C}^{\infty}$. Hence, the function defined by this integral is in $C^{n}(\alpha, 2 \pi+\alpha)$. We need only prove that the function $I(t)$ defined by the first integral of the equation above is in $C^{n}(\alpha, 2 \pi+\alpha)$. Notice that

$$
\begin{aligned}
I(t)= & \int_{t-2 \pi-\alpha}^{t-\alpha} F(t-s) \log |s| d s+\int_{t-\alpha}^{2 \pi+t-\alpha} F(2 \pi+t-s) \log |s| d s \\
& +\int_{2 \pi-t+\alpha}^{4 \pi-t+\alpha} F(s-2 \pi+t) \log |s| d s .
\end{aligned}
$$

Then it suffices to prove the following formula: for $t \in(\alpha, 2 \pi+\alpha)$,

$$
\begin{aligned}
\frac{d^{i} I}{d t^{i}}= & \int_{t-2 \pi-\alpha}^{t-\alpha} F^{(i)}(t-s) \log |s| d s+\int_{t-\alpha}^{2 \pi+t-\alpha} F^{(i)}(2 \pi+t-s) \log |s| d s \\
& +\int_{2 \pi-t+\alpha}^{4 \pi-t+\alpha} F^{(i)}(s-2 \pi+t) \log |s| d s+x_{i}(t), \quad i=0,1, \ldots, n
\end{aligned}
$$

where $x_{i}$ is some function in $C^{\infty}[\alpha, 2 \pi+\alpha]$. This formula holds trivially for $i=0$ with $x_{0}=0$. We assume that it holds for some integer $i$ and prove that it holds for 
$i+1$. Notice that for $t \in(\alpha, 2 \pi+\alpha)$,

$$
\begin{aligned}
& \frac{d}{d t} \int_{t-2 \pi-\alpha}^{t-\alpha} F^{(i)}(t-s) \log |s| d s \\
& \quad=\int_{t-2 \pi-\alpha}^{t-\alpha} F^{(i+1)}(t-s) \log |s| d s \\
& \quad+F^{(i)}(\alpha) \log |t-\alpha|-F^{(i)}(2 \pi+\alpha) \log |t-2 \pi-\alpha| \\
& \frac{d}{d t} \int_{t-\alpha}^{2 \pi+t-\alpha} F^{(i)}(2 \pi+t-s) \log |s| d s \\
& \quad \int_{t-\alpha}^{2 \pi+t-\alpha} F^{(i+1)}(2 \pi+t-s) \log |s| d s \\
& \quad+F^{(i)}(\alpha) \log |2 \pi+t-\alpha|-F^{(i)}(2 \pi+\alpha) \log |t-\alpha|
\end{aligned}
$$

and

$$
\begin{aligned}
& \frac{d}{d t} \int_{2 \pi-t+\alpha}^{4 \pi-t+\alpha} F^{(i)}(s-2 \pi+t) \log |s| d s \\
& =\int_{2 \pi-t+\alpha}^{4 \pi-t+\alpha} F^{(i+1)}(s-2 \pi+t) \log |s| d s \\
& \quad-F^{(i)}(2 \pi+\alpha) \log |4 \pi-t+\alpha|+F^{(i)}(\alpha) \log |2 \pi-t+\alpha| .
\end{aligned}
$$

Then, by the periodicity of $F$ and these identities, we have that for $t \in(\alpha, 2 \pi+\alpha)$,

$$
\begin{aligned}
\frac{d^{i+1} I}{d t^{i+1}}= & \int_{t-2 \pi-\alpha}^{t-\alpha} F^{(i+1)}(t-s) \log |s| d s+\int_{t-\alpha}^{2 \pi+t-\alpha} F^{(i+1)}(2 \pi+t-s) \log |s| d s \\
& +\int_{2 \pi-t+\alpha}^{4 \pi-t+\alpha} F^{(i+1)}(s-2 \pi+t) \log |s| d s+x_{i+1}(t)
\end{aligned}
$$

where

$$
x_{i+1}(t)=x_{i}^{\prime}(t)+F^{(i)}(\alpha) \log \left(\frac{2 \pi+t-\alpha}{4 \pi-t+\alpha}\right),
$$

which is in $C^{\infty}[\alpha, 2 \pi+\alpha]$. This completes the proof of the formula and the lemma as well.

Since (1.10) is a Fredholm integral equation of the second kind, we consider the following Fredholm integral equations in a more general setting that includes equation (1.10) as a special case:

$$
\phi(t)-\lambda \int_{a}^{b} k(t, s) \phi(s) d s=f(t), \quad a \leq t \leq b .
$$

The kernel in (1.11) takes the form

$$
\begin{aligned}
k(t, s)= & H_{1}(t, s) \log (|t-s|)+H_{2}(t, s) \log (|T-s+t|) \\
& +H_{3}(t, s) \log (|T-t+s|)+H_{4}(t, s),
\end{aligned}
$$

where $T=b-a$. Let $m \geq 1$ be an integer. We assume that

$$
\begin{gathered}
H_{1}, H_{4} \in C^{2 m}\left(\left\{(t, s):|t-s| \leq \frac{3}{2} T, t \in(-\infty, \infty)\right\}\right), \\
H_{2} \in C^{2 m}\left(\left\{(t, s):-2 T \leq s-t \leq \frac{5}{2} T, t \in(-\infty, \infty)\right\}\right),
\end{gathered}
$$


and

$$
H_{3} \in C^{2 m}\left(\left\{(t, s):-\frac{5}{2} T \leq s-t \leq 2 T, t \in(-\infty, \infty)\right\}\right)
$$

are chosen so that the kernel $k$ is periodic in both $t$ and $s$ with period $T$. In addition, we assume that $H_{1}(t, t), H_{2}(t, t), H_{3}(t, t)$, and $H_{4}(t, t)$ are periodic in $t$ with the same period $T$. The function $f$ on the right-hand side is also assumed to be periodic in $t$ with period $T$ and in $C^{2 m}(-\infty, \infty)$. We remark that a solution of equation (1.11) is also periodic with period $T$, since

$$
\begin{aligned}
\phi(a) & =\lambda \int_{a}^{b} k(a, s) \phi(s) d s+f(a) \\
& =\lambda \int_{a}^{b} k(a+T, s) \phi(s) d s+f(a+T) \\
& =\lambda \int_{a}^{b} k(b, s) \phi(s) d s+f(b) \\
& =\phi(b) .
\end{aligned}
$$

Clearly, equation (1.10) satisfies all conditions on (1.11) if $g$ in (1.2) is in $C^{2 m}(\Gamma)$.

Let $C_{T}[a, b]$ be the space of continuous periodic functions on $[a, b]$ with period $T$ with the uniform norm $\|\cdot\|$. Then $C_{T}[a, b]$ is a Banach space. We now define an operator $K: C_{T}[a, b] \rightarrow C_{T}[a, b]$ by

$$
(K \phi)(t)=\int_{a}^{b} k(t, s) \phi(s) d s \text { for } \phi \in C_{T}[a, b] .
$$

In operator notation, equation (1.11) can be written as

$$
\phi-\lambda K \phi=f .
$$

Clearly, $K$ is a compact operator in $C_{T}[a, b]$, with a weakly singular kernel. If $\lambda$ is not an eigenvalue of the operator $K$, then equation (1.11) has a unique solution in $C_{T}[a, b][1,2,3]$.

In general, the solution of equation (1.11) is as smooth as $f$ is in the interior of $(a, b)$, but may have mild singularity at the endpoints $a$ and $b$, namely, the derivative of $\phi$ may be unbounded at $a$ and $b$ (see [11]). However, as argued in [13], the periodicity property of $\phi$ ensures that $\phi$ has no singularity at either endpoint, and then $\phi$ is as smooth as $f$ is in $(-\infty, \infty)$. In fact, since $k, f$ and $\phi$ are all periodic with period $T$, the limits $a$ and $b$ in (1.11) can be replaced by $a^{\prime}$ and $b^{\prime}$ respectively, with $b^{\prime}-a^{\prime}=T$. In particular, choose a pair $a^{\prime}, b^{\prime}$ with $b^{\prime}-a^{\prime}=T$ such that $a \in\left(a^{\prime}, b^{\prime}\right)$ and replace $a$ and $b$ in (1.11) by $a^{\prime}$ and $b^{\prime}$, respectively. Then the solution $\phi$ of (1.11) is as smooth as $f$ is at $a$, since $a$ is an interior point of the interval $\left[a^{\prime}, b^{\prime}\right]$. Similarly, we prove that $\phi$ is as smooth as $f$ is at $b$. As a result, we conclude that $\phi$ is as smooth as $f$ is on $(-\infty, \infty)$.

In this paper, we derive an extrapolation scheme for the approximate solutions of (1.11) obtained by Nyström methods with a subdivision of the given partition. An asymptotic expansion for such approximate solutions is presented. The paper is organized as follows: In $\S 2$, we derive the Nyström method by using a quadrature formula of Sidi and Israeli, state the main theorem of this paper that gives an asymptotic expansion of approximate solutions, and derive the extrapolation scheme by using this asymptotic expansion. In $\S 3$, we prove two different convergence properties of the approximate operators. In $\S 4$, we give the proof for the 
main theorem stated in $\S 2$. In $\S 5$, some computational aspects of the Nyström method are considered and two numerical examples are presented to illustrate the theoretical estimates for the extrapolation scheme.

\section{NYSTRÖM SCHEME AND EXTRAPOLATION METHODS}

In this section, we present a Nyström scheme and its extrapolation method and state the main theorem of this paper. We first recall a known result of Sidi and Israeli that will be used to establish the Nyström method on the basis of which the extrapolation procedure is defined.

Let $s_{j}=a+j h, j=0,1, \ldots, n, h=(b-a) / n$, where $n$ is a positive integer. Let $t \in[a, b]$ be fixed. The following Theorem 2.1 can be found in $[12,13]$. Some early work on Euler-Maclaurin expansions for integrals with singularity may be found in $[8,9]$, and a general extrapolation method is discussed in [7].

Theorem 2.1 (Sidi and Israeli). Let $m>1$ be an integer. Let $t$ be one of the points in

$$
S_{n-1}:=\left\{s_{1}, s_{2}, \ldots, s_{n-1}\right\}
$$

Assume that $\hat{g} \in C^{2 m}[a, b]$. Let

$$
G(s)=|s-t|^{\beta} \log (|s-t|) \hat{g}(s), \quad \beta>-1 .
$$

Then

$$
\begin{aligned}
\int_{a}^{b} G(s) d s= & \frac{h}{2}\left(G\left(s_{0}\right)+G\left(s_{n}\right)\right)+h \sum_{j=1, s_{j} \neq t}^{n-1} G\left(s_{j}\right) \\
& +\sum_{\mu=1}^{m-1} \frac{B_{2 \mu}}{(2 \mu) !}\left[G^{(2 \mu-1)}(a)-G^{(2 \mu-1)}(b)\right] h^{2 \mu} \\
& -2 \sum_{\mu=0}^{m-1}\left[-\zeta^{\prime}(-\beta-2 \mu)+\zeta(-\beta-2 \mu) \log (h)\right] \frac{\hat{g}^{(2 \mu)}(t)}{(2 \mu) !} h^{2 \mu+\beta+1} \\
& +O\left(h^{2 m}\right), \quad h \rightarrow 0
\end{aligned}
$$

where $\zeta(\tau)$ denotes the Riemann zeta function defined for $\operatorname{Re} \tau>1$ by $\zeta(\tau)=$ $\sum_{n=1}^{\infty} n^{-\tau}$ and $B_{2 \mu}$ are the Bernoulli numbers.

Using this result, we develop a generalized Euler-Maclaurin formula on which our Nyström scheme and extrapolation method are based.

Theorem 2.2. Let $m>1$ be an integer and $t \in[a, b]$ be fixed. Assume that $g_{1}, g_{4} \in C^{2 m}\left[a-\frac{T}{2}, b+\frac{T}{2}\right], g_{2} \in C^{2 m}\left[a-\frac{T}{2}, b+\frac{3 T}{2}\right]$, and $g_{3} \in C^{2 m}\left[a-\frac{3 T}{2}, b+\frac{T}{2}\right]$. Let

$$
G(s)=\log (|s-t|) g_{1}(s)+\log (|T-s+t|) g_{2}(s)+\log (|T-t+s|) g_{3}(s)+g_{4}(s),
$$


and assume that $G$ is periodic with period $T$ on $\tilde{R}=(-\infty, \infty) \backslash\{t+k T\}_{k=-\infty}^{\infty}$. Then

$$
\begin{gathered}
\int_{a}^{b} G(s) d s=h \sum_{j \neq 0, a<t+j h \leq b} G(t+j h)+g_{1}(t) h \log \left(\frac{h}{2 \pi}\right)+h\left(g_{2}(t)+g_{3}(t)\right) \log (T) \\
+h g_{4}(t)+2 \sum_{\mu=1}^{m-1} \frac{\zeta^{\prime}(-2 \mu)}{(2 \mu) !} g_{1}^{(2 \mu)}(t) h^{2 \mu+1}+O\left(h^{2 m}\right), \quad h \rightarrow 0 .
\end{gathered}
$$

Proof. First, we prove that the theorem holds if $t \in S_{n-1}$. Denote

$$
\begin{gathered}
G_{1}(s)=\log (|t-s|) g_{1}(s), \\
G_{2}(s)=\log (|s-(T+t)|) g_{2}(s)
\end{gathered}
$$

and

$$
G_{3}(s)=\log (|s-(t-T)|) g_{3}(s)
$$

We rewrite

$$
\int_{a}^{b} \log (|T-s+t|) g_{2}(s) d s=\int_{a}^{b+T} G_{2}(s) d s-\int_{b}^{b+T} G_{2}(s) d s
$$

and

$$
\int_{a}^{b} \log (|T-t+s|) g_{3}(s) d s=\int_{a-T}^{b} G_{3}(s) d s-\int_{a-T}^{a} G_{3}(s) d s,
$$

so that the singular point of each integral is always within the lower and upper limits. Applying Theorem 2.1 with $\beta=0$ to each of the integrals $\int_{a}^{b} G_{1}(s) d s$, $\int_{a}^{b+T} G_{2}(s) d s, \int_{b}^{b+T} G_{2}(s) d s, \int_{a-T}^{b} G_{3}(s) d s$, and $\int_{a-T}^{a} G_{3}(s) d s$, and applying the usual Euler-Maclaurin formula to $\int_{a}^{b} g_{4}(s) d s$, we have

$$
\begin{aligned}
\int_{a}^{b} G(s) d s= & \frac{h}{2}\left(G\left(s_{0}\right)+G\left(s_{n}\right)\right) \\
& +h \sum_{j=1, s_{j} \neq t}^{n-1} G\left(s_{j}\right)+h\left(g_{2}(t)+g_{3}(t)\right) \log (T)+h g_{4}(t) \\
& +\sum_{\mu=1}^{m-1} \frac{B_{2 \mu}}{(2 \mu) !}\left[G^{(2 \mu-1)}(a)-G^{(2 \mu)-1)}(b)\right] h^{2 \mu} \\
& -2 \sum_{\mu=0}^{m-1}\left[-\zeta^{\prime}(-2 \mu)+\zeta(-2 \mu) \log (h)\right] \frac{g_{1}^{(2 \mu)}(t)}{(2 \mu) !} h^{2 \mu+1}+O\left(h^{2 m}\right) .
\end{aligned}
$$

By using the periodicity of $G$ and noticing that $\zeta(0)=-1 / 2, \zeta(-2 \mu)=0$ for $\mu=1,2, \ldots$, and $\zeta^{\prime}(0)=-1 / 2 \log (2 \pi)$, we have

$$
\begin{array}{rl}
\int_{a}^{b} G(s) d s=h \sum_{j=1, s_{j} \neq t}^{n} & G\left(s_{j}\right)+g_{1}(t) h \log \left(\frac{h}{2 \pi}\right) \\
& +h\left(g_{2}(t)+g_{3}(t)\right) \log (T)+h g_{4}(t) \\
& +2 \sum_{\mu=1}^{m-1} \frac{\zeta^{\prime}(-2 \mu)}{(2 \mu) !} g_{1}^{(2 \mu)}(t) h^{2 \mu+1}+O\left(h^{2 m}\right) .
\end{array}
$$


Since $t$ is one of the points in $S_{n-1}$, we have

$$
\sum_{j=1, s_{j} \neq t}^{n} G\left(s_{j}\right)=\sum_{j \neq 0, a<t+j h \leq b} G(t+j h) .
$$

Hence, for $t \in S_{n-1}$ we have established formula (2.1).

If $t \in[a, b] \backslash S_{n-1}$, we choose an interval $\left[a^{\prime}, b^{\prime}\right]$ with $b^{\prime}-a^{\prime}=T$ such that $t$ coincides with one of the points in

$$
S_{n-1}^{\prime}:=\left\{s_{j}^{\prime}: j=1,2, \ldots, n-1\right\}
$$

with $s_{j}^{\prime}=a^{\prime}+j h$. By the periodicity of $G$, we have

$$
\int_{a}^{b} G(s) d s=\int_{a^{\prime}}^{b^{\prime}} G(s) d s .
$$

Since $t \in[a, b]$, if $h \leq \frac{T}{2}$, we can choose $a^{\prime}, b^{\prime}$ such that $a-\frac{T}{2} \leq a^{\prime}, b^{\prime} \leq b+\frac{T}{2}$. Because we have proved in the last paragraph that formula (2.1) with $a$ and $b$ replaced by $a^{\prime}$ and $b^{\prime}$ holds for $t \in S_{n-1}^{\prime}$, applying the formula to the integral $\int_{a^{\prime}}^{b^{\prime}} G(s) d s$ gives

$$
\begin{aligned}
& \int_{a}^{b} G(s) d s= h \sum_{j \neq 0, a^{\prime}<t+j h \leq b^{\prime}} G(t+j h)+g_{1}(t) h \log \left(\frac{h}{2 \pi}\right) \\
&+h\left(g_{2}(t)+g_{3}(t)\right) \log (T)+h g_{4}(t) \\
&+2 \sum_{\mu=1}^{m-1} \frac{\zeta^{\prime}(-2 \mu)}{(2 \mu) !} g_{1}^{(2 \mu)}(t) h^{2 \mu+1}+O\left(h^{2 m}\right), h \rightarrow 0 .
\end{aligned}
$$

Again, by the periodicity of $G$, we have that

This completes our proof.

$$
\sum_{j \neq 0, a^{\prime}<t+j h \leq b^{\prime}} G(t+j h)=\sum_{j \neq 0, a<t+j h \leq b} G(t+j h) .
$$

In Theorem 2.2, we assume that $m>1$ in order to have a complete expansion as given above. If $m=1$, then it can be verified (see $[8,9]$ ) that the following asymptotic expansion holds:

$$
\begin{aligned}
\int_{a}^{b} G(s) d s= & h \sum_{\substack{j \neq 0, a<t+j h \leq b\\
}} G(t+j h)+g_{1}(t) h \log \left(\frac{h}{2 \pi}\right) \\
& +h\left(g_{2}(t)+g_{3}(t)\right) \log (T)+h g_{4}(t)+O\left(h^{2}\right), h \rightarrow 0 .
\end{aligned}
$$

We now use Theorem 2.2 to develop the Nyström scheme for solving equation (1.11). Let $K$ be the integral operator defined by (1.12) with $H_{i}, i=1,2,3,4$, given in $\S 1$ and $\phi$ the solution of equation (1.11). Applying Theorem 2.2 to $G(s)=$ $k(t, s) \phi(s)$ yields

$$
\begin{aligned}
(K \phi)(t)= & h \sum_{\substack{j \neq 0, a<t+j h \leq b\\
}} k(t, t+j h) \phi(t+j h)+H_{1}(t, t) \phi(t) h \log \left(\frac{h}{2 \pi}\right) \\
& +h\left(H_{2}(t, t)+H_{3}(t, t)\right) \log (T) \phi(t) \\
& +h H_{4}(t, t) \phi(t)+2 \sum_{\mu=1}^{m-1} \frac{\zeta^{\prime}(-2 \mu)}{(2 \mu) !} \frac{\partial^{2 \mu}}{\partial s^{2 \mu}}\left[H_{1}(t, s) \phi(s)\right]_{s=t} h^{2 \mu+1}+O\left(h^{2 m}\right) .
\end{aligned}
$$


For each positive integer $n$, we define an operator $K_{n}: C_{T}[a, b] \rightarrow C_{T}[a, b]$ by

$$
\begin{aligned}
\left(K_{n} \phi\right)(t)= & h \sum_{\substack{j \neq 0, a<t+j h \leq b\\
}} k(t, t+j h) \phi(t+j h)+H_{1}(t, t) \phi(t) h \log \left(\frac{h}{2 \pi}\right) \\
& +h\left(H_{2}(t, t)+H_{3}(t, t)\right) \log (T) \phi(t)+h H_{4}(t, t) \phi(t),
\end{aligned}
$$

where $h=(b-a) / n$. Then, $K_{n}$ approximates $K$ with truncation error

$$
\begin{aligned}
(K \phi)(t)= & \left(K_{n} \phi\right)(t)+2 \sum_{\mu=1}^{m-1} \frac{\zeta^{\prime}(-2 \mu)}{(2 \mu) !} \frac{\partial^{2 \mu}}{\partial s^{2 \mu}}\left[H_{1}(t, s) \phi(s)\right]_{s=t} h^{2 \mu+1} \\
& +O\left(h^{2 m}\right) \text { for } \phi \in C_{T}^{2 m}(-\infty, \infty),
\end{aligned}
$$

where we use $C_{T}^{2 m}(-\infty, \infty)$ to denote the space of $T$-periodic functions in $C^{2 m}(-\infty, \infty)$. Replacing $K$ by $K_{n}$ in equation (1.11) leads to the following approximate equation:

$$
\phi_{n}(t)-\lambda\left(K_{n} \phi_{n}\right)(t)=f(t), \quad t \in[a, b] .
$$

Equation (2.4) is called the Nyström scheme for solving equation (1.11), and a solution of (2.4) is called a Nyström solution for equation (1.11). This equation is the one we use to generate approximate solutions $\phi_{n}$ to the solution of (1.11). In the next section, we will show that the operators $K_{n}$ are uniformly bounded with respect to $n$. If $\left\|\lambda K_{n}\right\|<1$, then equation (2.4) has a unique solution and the solution is continuously dependent on the right-hand side. This condition can be weakened by assuming $\left\|(I-\lambda K)^{-1} \lambda\left(K-K_{n}\right) K_{n}\right\|<1$. Since our main interest of this paper is to study the asymptotic analysis for the solution of (2.4), we will simply assume, without further mentioning, that $\left(I-\lambda K_{n}\right)^{-1}$ exists and is uniformly bounded for sufficiently large $n$. More discussion about the computational issues of equation (2.4) will be given in $\S 5$.

We now state the main result of this paper, which gives an asymptotic expansion of the Nyström solution $\phi_{n}$. The proof of this theorem is given in $\S 4$.

Theorem 2.3. Let $\phi$ be the solution of equation (1.11) and $\phi_{n}$ the solution of equation (2.4). Suppose that $\left[\frac{\partial^{i}}{\partial s^{i}} H_{1}(t, s)\right]_{s=t}$ is a periodic function of $t$ with period $T$, for $i=1,2, \ldots, 2 m-2$. Then the following asymptotic expansion for $\phi_{n}$ holds:

$$
\phi_{n}(t)=\phi(t)-v_{3}(t) h^{3}-\sum_{q=5}^{2 m-1} v_{q}(t) h^{q}+O\left(h^{2 m}\right), \quad t \in[a, b],
$$

where $v_{q}$ are some functions independent of $h$.

We remark that a sufficient condition for $\left[\frac{\partial^{i}}{\partial s^{i}} H_{1}(t, s)\right]_{s=t}$ being periodic is that $H_{1}(t, s)$ is periodic in both $t$ and $s$.

Theorem 2.3 suggests the following extrapolation algorithm for $\phi_{n}$ : For each $t \in[a, b]$, define

$$
\begin{gathered}
\phi_{n, 0}(t)=\phi_{n}(t), \\
\phi_{n, 1}(t)=\frac{8 \phi_{2 n, 0}(t)-\phi_{n, 0}(t)}{7}
\end{gathered}
$$


and

$$
\phi_{n, \ell}(t)=\frac{2^{\ell+3} \phi_{2 n, \ell-1}(t)-\phi_{n, \ell-1}(t)}{2^{\ell+3}-1}, \quad \ell=2,3, \ldots .
$$

From Theorem 2.3, we obtain immediately the order of convergence for the $\ell$ th extrapolated approximate solutions $\phi_{n, \ell}$. This result is summarized in the following theorem.

Theorem 2.4. Suppose that the conditions of Theorem 2.3 hold. Then

$$
\phi_{n, \ell}(t)=\phi(t)+\sum_{j=\ell+4}^{2 m-1} v_{\ell, j}(t) h^{j}+O\left(h^{2 m}\right), t \in[a, b], \ell=1,2, \ldots,
$$

where $v_{\ell, j}$ are functions independent of $h$.

It follows from Theorem 2.4 that the first extrapolation increases the convergence order from 3 to 5 , and after the first step each extrapolation increases the convergence order by one.

\section{Convergence properties of $K_{n}$}

In this section, we study some properties of the approximate operators $K_{n}$ defined by (2.2), including pointwise convergence and uniform convergence of $\left(K_{n} \phi\right)(t)$ for $t \in[a, b]$. These results are needed for our further development, and they are of independent interest as well.

We first prove the pointwise convergence of $\left(K_{n} \phi\right)(t)$, for every $\phi \in C_{T}[a, b]$, in $[a, b]$. To do this, we introduce some additional notation and state a known result. Let $M$ designate the class of functions $\hat{g} \geq 0$ which are continuous and nondecreasing in $\left[a^{\prime}, b^{\prime}\right)$ and such that

$$
\lim _{t \rightarrow b^{\prime}-} \int_{a^{\prime}}^{t} \hat{g}(s) d s<\infty .
$$

Let $B M$ designate the class of functions $y$ that are continuous in $\left[a^{\prime}, b^{\prime}\right)$ and such that for each $y$ we can find a $\hat{g} \in M$ with $|y(s)| \leq \hat{g}(s)$ for $s \in\left[a^{\prime}, b^{\prime}\right)$. Define a sequence of quadrature formulas for a function $y$ by

$$
Q_{n}(y)=\sum_{k=1}^{n} w_{n k} y\left(s_{n k}\right)
$$

where

$$
a^{\prime} \leq s_{n n}<s_{n, n-1}<\cdots<s_{n 1}<s_{n 0}=b^{\prime} .
$$

The following theorem of Rabinowitz can be found in [6, p. 182].

Theorem 3.1. Suppose that

$$
\lim _{n \rightarrow \infty} Q_{n}(y)=\int_{a^{\prime}}^{b^{\prime}} y(s) d s
$$

for all $y \in C\left[a^{\prime}, b^{\prime}\right]$ and that there exists a constant $c>0$ such that

$$
\left|w_{n k}\right| \leq c\left(s_{n, k-1}-s_{n k}\right)
$$

for all sufficiently large $n$ and for all $k$ such that

$$
\left|s_{n k}-b^{\prime}\right|<\delta
$$

for some fixed $\delta>0$. Then equation (3.1) holds for all $y \in B M$. 
We are now ready to state and prove pointwise convergence of $\left(K_{n} \phi\right)(t)$.

Theorem 3.2. Let $\phi \in C_{T}[a, b]$. Then $\left(K_{n} \phi\right)(t)$ converges pointwise to $(K \phi)(t)$ for $t \in[a, b]$, as $n \rightarrow \infty$.

Proof. Denote the operators $\hat{K}_{n}$ for $\phi \in C_{T}[a, b]$, by

$$
\left(\hat{K}_{n} \phi\right)(t)=h \sum_{j \neq 0, a<t+j h \leq b} k(t, t+j h) \phi(t+j h) .
$$

Then, from equation (2.2) we have

$$
\begin{aligned}
\left(K_{n} \phi\right)(t)= & \left(\hat{K}_{n} \phi\right)(t)+H_{1}(t, t) \phi(t) h \log \left(\frac{h}{2 \pi}\right) \\
& +h\left(H_{2}(t, t)+H_{3}(t, t)\right) \log (T) \phi(t)+h H_{4}(t, t) \phi(t) .
\end{aligned}
$$

Since the last three terms vanish if $h \rightarrow 0$, it is sufficient to show that $\left(\hat{K}_{n} \phi\right)(t)$ converges to $(K \phi)(t)$ pointwise. By making use of the periodicity of $k$ and $\phi$, we rewrite

$$
(K \phi)(t)=\int_{t-T}^{t} k(t, s) \phi(s) d s
$$

Equivalently,

$$
\begin{aligned}
(K \phi)(t)= & \int_{t-T}^{t} \log (|t-s|) H_{1}(t, s) \phi(s) d s+\int_{t-T}^{t} \log (|T-s+t|) H_{2}(t, s) \phi(s) d s \\
& +\int_{t-T}^{t} \log (|T-t+s|) H_{3}(t, s) \phi(s) d s+\int_{t-T}^{t} H_{4}(t, s) \phi(s) d s .
\end{aligned}
$$

Now we rewrite $\left(\hat{K}_{n} \phi\right)(t)$, according to equation (3.2) by using the periodicity, as

$$
\left(\hat{K}_{n} \phi\right)(t)=h \sum_{j=1}^{n-1} k(t, t+j h-T) \phi(t+j h-T),
$$

that is,

$$
\begin{aligned}
\left(\hat{K}_{n} \phi\right)(t)= & h \sum_{j=1}^{n-1} H_{1}(t, t+j h-T) \log (|t-(t+j h-T)|) \phi(t+j h-T) \\
& +h \sum_{j=1}^{n-1} H_{2}(t, t+j h-T) \log (|T-(t+j h-T)+t|) \phi(t+j h-T) \\
& +h \sum_{j=1}^{n-1} H_{3}(t, t+j h-T) \log (|T-t+(t+j h-T)|) \phi(t+j h-T) \\
& +h \sum_{j=1}^{n-1} H_{4}(t, t+j h-T) \phi(t+j h-T) .
\end{aligned}
$$

Since the integrands of the second and fourth integrals on the right-hand side of equation $\left(3.2^{\prime}\right)$ are continuous on $[t-T, t]$, the second and fourth summations on the right-hand side of $\left(3.3^{\prime}\right)$ converge to the corresponding integrals, respectively. 
The integrand of the first integral on the right-hand side of $\left(3.2^{\prime}\right)$ has a singularity at $s=t$, and the first summation on the right-hand side of $\left(3.3^{\prime}\right)$ defines a quadrature formula $Q_{n}(y)$, where $y(s)=H_{1}(t, s) \log (|t-s|) \phi(s)$, for the first integral $\int_{t-T}^{t} y(s) d s$ on the right-hand side of $\left(3.2^{\prime}\right)$.It is clear that if the integrand of this integral is replaced by a continuous function, the corresponding quadrature converges to the integral. By Theorem 3.1, in order to show $Q_{n}(y) \rightarrow \int_{t-T}^{t} y(s) d s$, we need only prove $y \in B M$. If $T=b-a \leq 1$, then

$$
|y(s)| \leq \max _{t-T \leq s \leq t}\left|H_{1}(t, s) \phi(s)\right||\log (|t-s|)|,
$$

and the function on the right-hand side is in class $M$. If $T=b-a>1$, then

$$
|y(s)| \leq \max _{t-T \leq s \leq t}\left|H_{1}(t, s) \phi(s)\right| g_{t}(s)
$$

where

$$
g_{t}(s)= \begin{cases}\log (T) & \text { if } s \in[t-T, t-1] \\ |\log (|t-s|)|+\log (T) & \text { if } s \in(t-1, t)\end{cases}
$$

It can be shown that the function on the right-hand side is in class M. Hence in both cases, we conclude that $y(s)$ as a function of $s$ is in class $B M$. By Theorem 3.1, the first summation on the right-hand side of $\left(3.3^{\prime}\right)$ converges to the first integral on the right-hand of $\left(3.2^{\prime}\right)$.Notice that the third integral on the right-hand side of $\left(3.2^{\prime}\right)$ can be rewritten as

$$
\int_{-t}^{-t+T} \log (|T-t-s|) H_{3}(t,-s) \phi(-s) d s
$$

whose integrand has a singularity at $s=-t+T$, and the third summation on the right-hand side of $\left(3.3^{\prime}\right)$ can be rewritten as

$$
h \sum_{j=1}^{n-1} H_{3}(t,-(-t+T-j h)) \log (|T-t-(-t+T-j h)|) \phi(-(-t+T-j h)),
$$

which defines a quadrature $Q_{n}\left(y^{*}\right)$, where $y^{*}(s)=\log (|T-t-s|) H_{3}(t,-s) \phi(-s)$, for $\int_{-t}^{-t+T} y^{*}(s) d s$. As seen before, we need only show $y^{*} \in B M$ in order to conclude that $Q_{n}\left(y^{*}\right) \rightarrow \int_{-t}^{-t+T} y^{*}(s) d s$ and then in turn conclude that the third summation on the right-hand side of $\left(3.3^{\prime}\right)$ converges to the third integral on the right-hand side of $\left(3.2^{\prime}\right)$. If $T=b-a \leq 1$, then

$$
\left|y^{*}(s)\right| \leq \max _{-t \leq s \leq-t+T}\left|H_{3}(t,-s) \phi(-s)\right||\log (|T-t-s|)|
$$

and the function on the right-hand side is in class $M$. If $T=b-a>1$, then

$$
\left|y^{*}(s)\right| \leq \max _{-t \leq s \leq-t+T}\left|H_{3}(t,-s) \phi(-s)\right| g_{t}^{*}(s)
$$

where

$$
g_{t}^{*}(s)= \begin{cases}\log (T) & \text { if } s \in[-t,-t+T-1] \\ |\log (|T-t-s|)|+\log (T) & \text { if } s \in(-t+T-1,-t+T) .\end{cases}
$$

It can be shown that the function on the right-hand side is in class M. Hence, in both cases we conclude that $y^{*}(s)$ as a function of $s$ is in class $B M$. It follows from the above derivation that $\left(\hat{K}_{n} \phi\right)(t)$ converges to $(K \phi)(t)$ pointwise for $t \in[a, b]$. The proof is complete. 
We now establish the uniform convergence of $\left\{\left(K_{n} \phi\right)(t)\right\}$ for $t \in[a, b]$. To this end, we need the following three lemmas.

Lemma 3.3. Let $n$ be a positive integer. Then

$$
\sum_{j=1}^{n} \frac{1}{n}\left|\log \left(\frac{j}{n}\right)\right| \leq 1
$$

Proof. Since $-\log (s)$ is decreasing and nonnegative on $(0,1]$, we have

$$
\sum_{j=1}^{n} \frac{1}{n}\left|\log \left(\frac{j}{n}\right)\right|=\sum_{j=1}^{n} \frac{1}{n}\left(-\log \left(\frac{j}{n}\right)\right) \leq \int_{0}^{1}-\log (s) d s=1 .
$$

For any positive integer $n$, we rewrite the operators $K_{n}$, using the periodicity property of $k$ and $\phi$, in the following way:

$$
\begin{aligned}
\left(K_{n} \phi\right)(t)= & h \sum_{j=1}^{n-1} k(t, t+j h) \phi(t+j h)+H_{1}(t, t) \phi(t) h \log \left(\frac{h}{2 \pi}\right) \\
& +h\left(H_{2}(t, t)+H_{3}(t, t)\right) \log (T) \phi(t)+h H_{4}(t, t) \phi(t) .
\end{aligned}
$$

Lemma 3.4. The sequence $\left\{\left\|K_{n}\right\|\right\}$ is bounded.

Proof. By equation (3.5), there holds, for all $t \in[a, b]$,

$$
\begin{aligned}
\left|\left(K_{n} \phi\right)(t)\right| \leq & \sum_{j=1}^{n-1}|h k(t, t+j h)|\|\phi\|+h\left|\log \left(\frac{h}{2 \pi}\right)\right| M_{1}\|\phi\| \\
& +h\left(M_{2}+M_{3}\right)|\log (T)|\|\phi\|+h M_{4}\|\phi\|
\end{aligned}
$$

where

$$
M_{j}=\max _{|t-s| \leq T, t \in[a, b]}\left|H_{j}(t, s)\right|, \quad j=1,2,3,4
$$

Since

$$
s \log \left(\frac{s}{2 \pi}\right) \rightarrow 0 \quad \text { as } s \rightarrow 0^{+}
$$

and $h=\frac{b-a}{n}$, there exists a positive constant $C_{1}$ such that $h\left|\log \left(\frac{h}{2 \pi}\right)\right| \leq C_{1}$ for all $n$. Thus,

$$
\begin{aligned}
\left|\left(K_{n} \phi\right)(t)\right| \leq\left(\sum_{j=1}^{n-1}|h k(t, t+j h)|+C_{1} M_{1}\right. \\
\left.\quad+\left(M_{2}+M_{3}\right)|\log (T)|(b-a)+M_{4}(b-a)\right)\|\phi\| .
\end{aligned}
$$


Using the expression for $k(t, t+j h)$, we conclude

$$
\begin{aligned}
\sum_{j=1}^{n-1}|h k(t, t+j h)| \leq & M_{1} \sum_{j=1}^{n-1}|h \log (j h)|+M_{2} \sum_{j=1}^{n-1}|h \log (|T-j h|)| \\
& +M_{3} \sum_{j=1}^{n-1}|h \log (|T+j h|)|+M_{4} \\
\leq & \left(M_{1}+M_{2}\right) \sum_{j=1}^{n-1}|h \log (j h)|+M_{3} \sum_{j=1}^{n-1}|h \log (T+j h)|+M_{4}
\end{aligned}
$$

By Lemma 3.3, we have the following estimate

$$
\begin{aligned}
\sum_{j=1}^{n-1} h|\log (j h)| & \leq(b-a)\left[|\log (b-a)|+\sum_{j=1}^{n-1} \frac{1}{n}\left|\log \left(\frac{j}{n}\right)\right|\right] \\
& \leq(b-a)[|\log (b-a)|+1]
\end{aligned}
$$

and thus $\sum_{j=1}^{n-1} h|k(t, t+j h)|$ is bounded by a constant. This implies that $\left\|K_{n} \phi\right\| \leq$ $C\|\phi\|$ for some constant $C>0$ and for all $n$.

Lemma 3.5. Let $\phi \in C_{T}[a, b]$. Then $\left(K_{n} \phi\right)(t)$ is equicontinuous on $[a, b]$, that is, for every $\epsilon>0$, there exists $\delta>0$ such that

$$
\left|\left(K_{n} \phi\right)(t)-\left(K_{n} \phi\right)(s)\right|<\epsilon
$$

for all $n$ and all $t, s \in[a, b]$ with $|t-s|<\delta$.

Proof. If $\phi=0$, then the statement of the lemma holds trivially. Assume $\phi \neq 0$. Again, by using equation (3.5), we have

$$
\begin{aligned}
\left(K_{n} \phi\right)(t)-\left(K_{n} \phi\right)(s)= & h \sum_{j=1}^{n-1}[k(t, t+j h)-k(s, s+j h)] \phi(s+j h) \\
& +h \sum_{j=1}^{n-1} k(t, t+j h)[\phi(t+j h)-\phi(s+j h)] \\
& +h \log \left(\frac{h}{2 \pi}\right)\left[H_{1}(t, t) \phi(t)-H_{1}(s, s) \phi(s)\right] \\
& +h\left[H_{2}(t, t) \phi(t)-H_{2}(s, s) \phi(s)\right] \log (T) \\
& +h\left[H_{3}(t, t) \phi(t)-H_{3}(s, s) \phi(s)\right] \log (T) \\
& +h\left[H_{4}(t, t) \phi(t)-H_{4}(s, s) \phi(s)\right] .
\end{aligned}
$$

Let $\epsilon>0$. Since $H_{4}(t, s) \phi(s), H_{3}(t, s) \phi(s), H_{2}(t, s) \phi(s)$ and $H_{1}(t, s) \phi(s)$ are uniformly continuous on $[a, b] \times[a, b]$, there exists $\delta_{1}>0$ such that, whenever $|t-s|<\delta_{1}$, 
we have

$$
\begin{gathered}
\left|H_{4}(t, t) \phi(t)-H_{4}(s, s) \phi(s)\right|<\frac{\epsilon}{6(b-a)}, \\
\left|H_{3}(t, t) \phi(t)-H_{3}(s, s) \phi(s)\right|<\frac{\epsilon}{6 \log (T)(b-a)}, \\
\left|H_{2}(t, t) \phi(t)-H_{2}(s, s) \phi(s)\right|<\frac{\epsilon}{6 \log (T)(b-a)}
\end{gathered}
$$

and

$$
\left|H_{1}(t, t) \phi(t)-H_{1}(s, s) \phi(s)\right|<\frac{\epsilon}{6 C_{1}}
$$

where $C_{1}$ is a bound for $h\left|\log \left(\frac{h}{2 \pi}\right)\right|$ given in the proof of Lemma 3.4. It follows that

$$
\begin{gathered}
h\left|H_{4}(t, t) \phi(t)-H_{4}(s, s) \phi(s)\right|<\frac{\epsilon}{6}, \\
h\left|H_{3}(t, t) \phi(t)-H_{3}(s, s) \phi(s)\right| \log (T)<\frac{\epsilon}{6}, \\
h\left|H_{2}(t, t) \phi(t)-H_{2}(s, s) \phi(s)\right| \log (T)<\frac{\epsilon}{6}
\end{gathered}
$$

and

$$
\left|H_{1}(t, t) \phi(t)-H_{1}(s, s) \phi(s)\right| h \log \left(\frac{h}{2 \pi}\right)<\frac{\epsilon}{6}
$$

By inequality (3.6) and Lemma 3.3, $\sum_{j=1}^{n-1} h|\log (j h)|$ is bounded for all $n$. Let $C_{2}$ be its bound. Since $H_{1}(t, s), H_{2}(t, s), H_{3}(t, s)$ and $H_{4}(t, s)$ are uniformly continuous on $\{(t, s):|t-s| \leq T, t \in[a, b]\}$, there exists $\delta_{2}>0$ such that, whenever $|t-s|<\delta_{2}$, we have

$$
\begin{gathered}
\left|H_{1}(t, t+j h)-H_{1}(s, s+j h)\right|<\frac{\epsilon}{24\|\phi\| C_{2}}, \\
\left|H_{2}(t, t+j h)-H_{2}(s, s+j h)\right|<\frac{\epsilon}{24\|\phi\| C_{2}}, \\
\left|H_{3}(t, t+j h)-H_{3}(s, s+j h)\right|<\frac{\epsilon}{24\|\phi\| \max _{0 \leq s \leq T}|\log (T+s)|}
\end{gathered}
$$

and

$$
\left|H_{4}(t, t+j h)-H_{4}(s, s+j h)\right|<\frac{\epsilon}{24(b-a)\|\phi\|} .
$$


Then

$$
\begin{aligned}
\sum_{j=1}^{n-1} h \mid & k(t, t+j h)-k(s, s+j h) \mid \\
\leq & \sum_{j=1}^{n-1} h\left|H_{1}(t, t+j h)-H_{1}(s, s+j h)\right||\log (j h)| \\
& +\sum_{j=1}^{n-1} h\left|H_{2}(t, t+j h)-H_{2}(s, s+j h)\right||\log (|T-j h|)| \\
& +\sum_{j=1}^{n-1} h\left|H_{3}(t, t+j h)-H_{3}(s, s+j h)\right||\log (|T+j h|)| \\
& +\sum_{j=1}^{n-1} h\left|H_{4}(t, t+j h)-H_{4}(s, s+j h)\right| \\
\leq & \sum_{j=1}^{n-1} h|\log (j h)| \frac{2 \epsilon}{24\|\phi\| C_{2}}+\frac{2 \epsilon}{24\|\phi\|} \\
\leq & \frac{\epsilon}{6\|\phi\|} .
\end{aligned}
$$

By the proof of Lemma 3.4, $\sum_{j=1}^{n-1} h|k(t, t+j h)|$ is bounded. Assume it is bounded by $C_{3}$. Since $\phi$ is uniformly continuous on $[a, b]$ and periodic, there exists $\delta_{3}>0$ such that for all $j$ and all $h>0$

$$
|\phi(t+j h)-\phi(s+j h)|<\frac{\epsilon}{6 C_{3}}
$$

whenever $|t-s|<\delta_{3}$. Let $\delta=\min \left\{\delta_{1}, \delta_{2}, \delta_{3}\right\}$. It follows from the above derivation that

$$
\left|\left(K_{n} \phi\right)(t)-\left(K_{n} \phi\right)(s)\right|<\epsilon
$$

whenever $|t-s|<\delta$.

Theorem 3.6. Let $\phi \in C_{T}[a, b]$. Then $\left(K_{n} \phi\right)(t)$ converges uniformly to $(K \phi)(t)$ on $[a, b]$, as $n \rightarrow \infty$.

Proof. Let $\epsilon>0$. Since $\left\{\left(K_{n} \phi\right)(t)\right\}$ is equicontinuous on $[a, b]$, there exists $\delta>0$ such that

$$
\left|\left(K_{n} \phi\right)(t)-\left(K_{n} \phi\right)(s)\right|<\epsilon / 3 \text { whenever } t, s \in[a, b],|t-s|<\delta, \text { for all } n .
$$

Let $a=t_{0}<t_{1}<\cdots<t_{m}=b$ be a fixed partition of $[a, b]$ with $t_{j}-t_{j-1}<\delta$ for $j=1,2, \ldots, m$. By assumption, $\left(K_{n} \phi\right)\left(t_{j}\right)$ converges to $(K \phi)\left(t_{j}\right)$ for each $j$. It follows that there exists $N>0$ such that

$$
\left|\left(K_{n} \phi\right)\left(t_{j}\right)-\left(K_{n+p} \phi\right)\left(t_{j}\right)\right|<\epsilon / 3 \text { whenever } n>N, p=1,2, \ldots, j=1,2, \ldots, m \text {. }
$$

Notice that for $t \in[a, b]$ we have $t \in\left[t_{j-1}, t_{j}\right]$ for some $j$ and thus $t_{j}-t<\delta$. Hence,

$\left|\left(K_{n} \phi\right)(t)-\left(K_{n+p} \phi\right)(t)\right| \leq\left|\left(K_{n} \phi\right)(t)-\left(K_{n} \phi\right)\left(t_{j}\right)\right|+\left|\left(K_{n} \phi\right)\left(t_{j}\right)-\left(K_{n+p} \phi\right)\left(t_{j}\right)\right|$ $+\left|\left(K_{n+p} \phi\right)\left(t_{j}\right)-\left(K_{n+p} \phi\right)(t)\right|<\epsilon$ 
whenever $n>N$ and $p=1,2, \ldots$ Hence,

$$
\left\|K_{n} \phi-K_{n+p} \phi\right\|<\epsilon
$$

whenever $n>N$ and $p=1,2, \ldots$. Consequently, $\left\{K_{n} \phi\right\}$ is a Cauchy sequence in $C_{T}[a, b]$. Since $C_{T}[a, b]$ is complete, $\left(K_{n} \phi\right)(t)$ converges uniformly to a function in $C_{T}[a, b]$. Again, since $\left(K_{n} \phi\right)(t)$ converges to $(K \phi)(t)$ pointwise, $\left(K_{n} \phi\right)(t)$ converges to $(K \phi)(t)$ uniformly.

As a direct consequence of Theorem 3.6, we show that if $\left(I-\lambda K_{n}\right)^{-1}$ exists and is uniformly bounded for sufficiently large $n$ on $C_{T}[a, b]$, then the unique solution $\phi_{n}$ of (2.4) converges to the unique solution $\phi$ of (1.11). In addition, suppose that $H_{1}, H_{2}, H_{3}, H_{4}$, and $f$ satisfy the assumption of this paper with $m=2$. Then, $\left\|\phi-\phi_{n}\right\| \leq C h^{3}$, where $C$ is a constant independent of $n$. To see this, we note from (1.11) and (2.4) that

$$
\phi-\phi_{n}=\lambda\left(K-K_{n}\right) \phi+\lambda K_{n}\left(\phi-\phi_{n}\right)
$$

Thus,

$$
\phi-\phi_{n}=\left(I-\lambda K_{n}\right)^{-1} \lambda\left(K-K_{n}\right) \phi
$$

By Theorem 3.6, we have

$$
\left\|\phi-\phi_{n}\right\| \leq C_{1} \lambda\left\|K \phi-K_{n} \phi\right\| \rightarrow 0 \text { as } n \rightarrow \infty .
$$

If the additional assumptions are imposed, using (2.3) with $m=2$, we find $\left\|\phi-\phi_{n}\right\|=O\left(h^{3}\right)$.

\section{Proof of Theorem 2.3}

In this section, we present the proof of Theorem 2.3.

Proof of Theorem 2.3. To prove the theorem, we show that there exist periodic functions $v_{q} \in C^{2\left(m-\left\lfloor\frac{q}{2}\right\rfloor\right)}(-\infty, \infty)$ with period $T$ such that

$$
\eta_{n}(t)=\phi(t)-\phi_{n}(t)+\sum_{q=3}^{2 m-1} v_{q}(t) h^{q}
$$

is of $O\left(h^{2 m}\right)$, where $\lfloor s\rfloor$ denotes the largest integer not greater than $s$. In the course of proving this, we shall construct the functions $v_{q}$.

Applying the operators $I-\lambda K_{n}$ to the both sides of (4.1) gives

$$
\left[\left(I-\lambda K_{n}\right) \eta_{n}\right](t)=\left[\left(I-\lambda K_{n}\right) \phi\right](t)-\left[\left(I-\lambda K_{n}\right) \phi_{n}\right](t)+\sum_{q=3}^{2 m-1}\left[\left(I-\lambda K_{n}\right) v_{q}\right](t) h^{q}
$$

Since $\phi \in C_{T}^{2 m}(-\infty, \infty), v_{q} \in C_{T}^{2\left(m-\left\lfloor\frac{q}{2}\right\rfloor\right)}(-\infty, \infty)$ if $q<2 m-2$, we have asymptotic expansions for $\left(I-\lambda K_{n}\right) \phi$ and $\left(I-\lambda K_{n}\right) v_{q}$ for $q<2 m-2$ in the form of (2.3). In addition, since $v_{2 m-1}, v_{2 m-2} \in C_{T}^{2}(-\infty, \infty)$, we have

$$
\left[\left(I-\lambda K_{n}\right) v_{q}\right](t)=\left[(I-\lambda K) v_{q}\right](t)+O\left(h^{2}\right), q=2 m-1,2 m-2 .
$$

Using the asymptotic expansions mentioned above and the relation

$$
[(I-\lambda K) \phi](t)=\left[\left(I-\lambda K_{n}\right) \phi_{n}\right](t)=f(t),
$$


we find that the right-hand side of (4.2) becomes

$$
\begin{aligned}
& -2 \sum_{\mu=1}^{m-1} \frac{\zeta^{\prime}(-2 \mu)}{(2 \mu) !} \frac{\partial^{2 \mu}}{\partial s^{2 \mu}}\left[H_{1}(t, s) \phi(s)\right]_{s=t} h^{2 \mu+1}+\sum_{q=3}^{2 m-1}\left[(I-\lambda K) v_{q}\right](t) h^{q} \\
& -2 \sum_{q=3}^{2 m-4} \sum_{\mu=1}^{m-\left\lfloor\frac{q}{2}\right\rfloor-1} \frac{\zeta^{\prime}(-2 \mu)}{(2 \mu) !} \frac{\partial^{2 \mu}}{\partial s^{2 \mu}}\left[H_{1}(t, s) v_{q}(s)\right]_{s=t} h^{2 \mu+q+1}+O\left(h^{2 m}\right) .
\end{aligned}
$$

Rearranging the terms in the third summation of the above quantity with respect to the order of $h$, we rewrite this summation as

$$
\begin{aligned}
& -2 \sum_{q=3}^{m-1} \sum_{\mu=1}^{q-2} \frac{\zeta^{\prime}(2 \mu+2-2 q)}{(2 q-2 \mu-2) !} \frac{\partial^{2(q-\mu-1)}}{\partial s^{2(q-\mu-1)}}\left[H_{1}(t, s) v_{2 \mu+1}(s)\right]_{s=t} h^{2 q} \\
& -2 \sum_{q=3}^{m-1} \sum_{\mu=2}^{q-1} \frac{\zeta^{\prime}(2 \mu-2 q)}{(2 q-2 \mu) !} \frac{\partial^{2(q-\mu)}}{\partial s^{2(q-\mu)}}\left[H_{1}(t, s) v_{2 \mu}(s)\right]_{s=t} h^{2 q+1}+O\left(h^{2 m}\right) .
\end{aligned}
$$

Therefore, the right-hand side of equation (4.2) becomes

$$
\begin{aligned}
\sum_{q=1}^{2}\left\{\left[(I-\lambda K) v_{2 q+1}\right](t)-\frac{2 \zeta^{\prime}(-2 q)}{(2 q) !} \frac{\partial^{2 q}}{\partial s^{2 q}}\left[H_{1}(t, s) \phi(s)\right]_{s=t}\right\} h^{2 q+1} & +\left[(I-\lambda K) v_{4}\right](t) h^{4} \\
& +\sum_{q=3}^{m-1}\left\{\left[(I-\lambda K) v_{2 q+1}\right)(t)-\frac{2 \zeta^{\prime}(-2 q)}{(2 q) !} \frac{\partial^{2 q}}{\partial s^{2 q}}\left[H_{1}(t, s) \phi(s)\right]_{s=t}\right. \\
& \left.-2 \sum_{\mu=2}^{q-1} \frac{\zeta^{\prime}(2 \mu-2 q)}{(2 q-2 \mu) !} \frac{\partial^{2(q-\mu)}}{\partial s^{2(q-\mu)}}\left[H_{1}(t, s) v_{2 \mu}(s)\right]_{s=t}\right\} h^{2 q+1} \\
& +\sum_{q=3}^{m-1}\left\{\left[(I-\lambda K) v_{2 q}\right](t)\right. \\
& +O\left(h^{2 m}\right) .
\end{aligned}
$$

We now choose $v_{q}(t)$ to be the solutions of the following integral equations:

$$
\begin{aligned}
{\left[(I-\lambda K) v_{2 q+1}\right](t)=} & 2 \frac{\zeta^{\prime}(-2 q)}{(2 q) !} \frac{\partial^{2 q}}{\partial s^{2 q}}\left[H_{1}(t, s) \phi(s)\right]_{s=t}, \quad q=1,2, \\
& {\left[(I-\lambda K) v_{4}\right](t)=0 } \\
{\left[(I-\lambda K) v_{2 q+1}\right](t)=} & \frac{2 \zeta^{\prime}(-2 q)}{(2 q) !} \frac{\partial^{2 q}}{\partial s^{2 q}}\left[H_{1}(t, s) \phi(s)\right]_{s=t} \\
+ & 2 \sum_{\mu=2}^{q-1} \frac{\zeta^{\prime}(2 \mu-2 q)}{(2 q-2 \mu) !} \frac{\partial^{2(q-\mu)}}{\partial s^{2(q-\mu)}}\left[H_{1}(t, s) v_{2 \mu}(s)\right]_{s=t} \\
q & =3, \ldots, m-1
\end{aligned}
$$


and

$$
\begin{gathered}
{\left[(I-\lambda K) v_{2 q}\right](t)=2 \sum_{\mu=1}^{q-2} \frac{\zeta^{\prime}(2 \mu+2-2 q)}{(2 q-2 \mu-2) !} \frac{\partial^{2(q-\mu-1)}}{\partial s^{2(q-\mu-1)}}\left[H_{1}(t, s) v_{2 \mu+1}(s)\right]_{s=t},} \\
q=3, \ldots, m-1 .
\end{gathered}
$$

Each of these equations has a unique solution. In particular, $v_{4}=0$. The functions $v_{q}$ are defined recursively by the solutions of the above equations. The periodicity of $\left[\frac{\partial^{i}}{\partial s^{i}} H_{1}(t, s)\right]_{s=t}$ implies that the functions on the right-hand side of the above equations are all periodic and so are the solutions. By the assumptions on $\phi$ and $H_{1}$, we conclude $v_{q} \in C_{T}^{2\left(m-\left\lfloor\frac{q}{2}\right\rfloor\right)}(-\infty, \infty)$. Once these functions are chosen, we have

$$
\left[\left(I-\lambda K_{n}\right) \eta_{n}\right](t)=O\left(h^{2 m}\right) .
$$

By assumption, there exists a constant $C$ such that $\left\|\left(I-\lambda K_{n}\right)^{-1}\right\| \leq C$, and thus $\eta_{n}(t)=O\left(h^{2 m}\right)$. The proof is complete.

\section{Computational aspects AND nUmerical EXAMPles}

In this section, we consider some computational aspects of the approximate equation (2.4) and present two examples to illustrate the accelerated convergence of extrapolation method.

Notice that the definition of $K_{n}$ in equation (2.4) is unconventional. In conventional quadrature schemes for integral equations with continuous kernels (see [1]), $\left(K_{n} \phi\right)(t)$ can be expressed as a combination of $\phi\left(s_{k}\right)$, and the coefficients may depend on $t$. In other words, $K_{n}$ maps a vector $\left(\phi\left(s_{1}\right), \ldots, \phi\left(s_{n}\right)\right)$ in $R^{n}$ to a continuous function. However, in the current case, $\left(K_{n} \phi\right)(t)$ is defined in terms of $\phi(t+j h), a<t+j h \leq b$. The function $\left(K_{n} \phi\right)(t)$ not only depends on $n$ values of $\phi$ at $n$ nodes, but also depends on the values of $\phi$ at every point in $[a, b]$.

To solve equation (2.4), we let $t=s_{i}$ for $i=1,2, \ldots, n$ in the equation. Since the extrapolation method will use two approximate solutions corresponding to different stepsizes $h$, we denote $\phi_{i}^{h}=\phi_{n}\left(s_{i}\right)$. In views of the periodicity of $k, \phi$ and $f$, if $\phi_{n}$ is a solution of equation $(2.4)$, then $\left(\phi_{1}^{h}, \ldots, \phi_{n}^{h}\right)$ satisfies the following algebraic equations:

$$
\begin{aligned}
& {\left[1-\lambda h \log \left(\frac{h}{2 \pi}\right) H_{1}\left(s_{i}, s_{i}\right)-\lambda h\left(H_{2}\left(s_{i}, s_{i}\right)+H_{3}\left(s_{i}, s_{i}\right)\right) \log (T)-\lambda h H_{4}\left(s_{i}, s_{i}\right)\right] \phi_{i}^{h}} \\
& \quad-\lambda h \sum_{j \neq i, j=1}^{n} k\left(s_{i}, s_{j}\right) \phi_{j}^{h}=f\left(s_{i}\right), i=1,2, \ldots, n .
\end{aligned}
$$

This system of linear equations is called the discrete Nyström method, and the solution of the system is called the discrete Nyström solution for (1.11). Clearly, if the system (5.1) has a unique solution $\left(\phi_{1}^{h}, \ldots, \phi_{n}^{h}\right)$, then $\phi_{i}^{h}=\phi_{n}\left(s_{i}\right), i=$ $1,2, \ldots, n$. In fact, it can be shown that if equation (2.4) has a unique solution $\phi_{n}$ for any continuous periodic right-hand side $f$, then the linear system (5.1) has a unique solution. To see this, we specialize the equation (2.4) at the nodes $s_{i}$ and we conclude that (5.1) has a solution for any right-hand side. This implies that the coefficient matrix of the system must be of full rank. Since it is a square matrix, it is nonsingular. However, by assumption, for a sufficiently large $n$, equation (2.4) 
has a unique solution. Hence, we conclude that for a sufficiently large $n$, the linear system (5.1) has a unique solution $\left(\phi_{1}^{h}, \ldots, \phi_{n}^{h}\right)$ and $\phi_{i}^{h}=\phi_{n}\left(s_{i}\right)$. Some points of the above discussion were motivated by [14].

Upon solving the linear system (5.1), we obtain the values of $\phi_{n}$ at the given nodes $s_{i}$. Thus, by using the extrapolation scheme described in $\S 2$, we obtain the discrete extrapolated approximate solutions, which have a higher order of convergence.

We next consider two examples.

Example 1. Consider the boundary value problem

$$
\begin{gathered}
\triangle u(P)=0, P \in D, \\
\frac{\partial u(P)}{\partial n_{P}}=-u(P)+g(P), \quad P \in \Gamma:=\partial D,
\end{gathered}
$$

where $g(P)=1$ and $D$ is the region

$$
\left(\frac{x}{a}\right)^{2}+\left(\frac{y}{b}\right)^{2}<1
$$

with $(a, b)=(1,2)$. It is not hard to verify that $u(x, y)=1$ is the unique solution of this problem. We will use our methods to obtain numerical solutions to this problem. Let

$$
x=\cos t, \quad y=2 \sin t, \quad 0 \leq t \leq 2 \pi .
$$

With this parametrization, the unit exterior normal vector of $\Gamma$ at $t$ is

$$
\left(\frac{2 x}{\sqrt{1+3 x^{2}}}, \frac{y}{2 \sqrt{1+3 x^{2}}}\right)=\left(\frac{2 \cos t}{\sqrt{4 \cos ^{2} t+\sin ^{2} t}}, \frac{\sin t}{\sqrt{4 \cos ^{2} t+\sin ^{2} t}}\right) \text {. }
$$

Since $\mathbf{r}(t)=(\cos t, 2 \sin t)$, we have $\left|\mathbf{r}^{\prime}(t)\right|=\sqrt{1+3 \cos ^{2} t} \neq 0$. Hence,

$$
\begin{aligned}
k(t, s)= & \frac{1}{\pi} \sqrt{1+3 \cos ^{2} s}(\log |t-s|+\log |2 \pi-s+t|+\log |2 \pi-t+s| \\
& \left.+\log \frac{\sqrt{(\cos t-\cos s)^{2}+4(\sin t-\sin s)^{2}}}{|t-s|(2 \pi-s+t)(2 \pi-t+s)}\right) \\
& +\frac{1}{\pi} \frac{2 \cos s(\cos s-\cos t)+\sin s(2 \sin s-2 \sin t)}{(\cos s-\cos t)^{2}+(2 \sin s-2 \sin t)^{2}} \\
= & \frac{1}{\pi} \sqrt{1+3 \cos ^{2} s} \log \sqrt{4 \sin ^{2} \frac{(t-s)}{2}+3(\sin t-\sin s)^{2}} \\
& +\frac{1}{\pi} \frac{4 \sin ^{2} \frac{t-s}{2}}{4 \sin ^{2} \frac{t-s}{2}+3(\sin t-\sin s)^{2}} .
\end{aligned}
$$

The formula given in Theorem 2.2 is used to calculate the integral in the righthand side of (1.10) and the discrete Nyström method (5.1) is used to compute approximate solutions of (1.10). The following two tables give the error of the approximate solutions using different stepsizes $h$ and of the extrapolated solutions, respectively. In Table 1 , we use $e_{i}=u\left(\mathbf{r}\left(t_{i}\right)\right)-\phi_{i}^{h}$ to denote the error of the Nyström solutions corresponding to the specified $h$. The rate of convergence guaranteed by Theorem 2.3 is of order 3 . 
TABLE 1. Errors of the Nyström solutions

\begin{tabular}{|c|c|c|c|c|c|}
\hline$t_{i}$ & $e_{i}$ with $h=\frac{2 \pi}{10}$ & $e_{i}$ with $h=\frac{2 \pi}{20}$ & rate & $e_{i}$ with $h=\frac{2 \pi}{40}$ & rate \\
\hline 0.628319 & $0.121881 \mathrm{D}-02$ & $0.131313 \mathrm{D}-03$ & 3.21 & $0.162984 \mathrm{D}-04$ & 3.01 \\
1.256637 & $-0.241312 \mathrm{D}-02$ & $-0.350908 \mathrm{D}-03$ & 2.78 & $-0.439971 \mathrm{D}-04$ & 3.00 \\
1.884956 & $-0.241325 \mathrm{D}-02$ & $-0.350658 \mathrm{D}-03$ & 2.78 & $-0.442431 \mathrm{D}-04$ & 2.99 \\
2.513274 & $0.121870 \mathrm{D}-02$ & $0.131397 \mathrm{D}-03$ & 3.21 & $0.162478 \mathrm{D}-04$ & 3.02 \\
3.141593 & $0.163276 \mathrm{D}-02$ & $0.189617 \mathrm{D}-03$ & 3.11 & $0.236295 \mathrm{D}-04$ & 3.00 \\
3.769911 & $0.121862 \mathrm{D}-02$ & $0.132229 \mathrm{D}-03$ & 3.20 & $0.171674 \mathrm{D}-04$ & 2.95 \\
4.398230 & $-0.241311 \mathrm{D}-02$ & $-0.351662 \mathrm{D}-03$ & 2.78 & $-0.435820 \mathrm{D}-04$ & 3.01 \\
5.026548 & $-0.241343 \mathrm{D}-02$ & $-0.351146 \mathrm{D}-03$ & 2.78 & $-0.437735 \mathrm{D}-04$ & 3.00 \\
5.654867 & $0.121874 \mathrm{D}-02$ & $0.131003 \mathrm{D}-03$ & 3.22 & $0.163326 \mathrm{D}-04$ & 3.00 \\
6.283185 & $0.163256 \mathrm{D}-02$ & $0.189412 \mathrm{D}-03$ & 3.11 & $0.236443 \mathrm{D}-04$ & 3.00 \\
\hline
\end{tabular}

In Table 2, we list the error of the extrapolated solutions by using the extrapolation algorithm described in $\S 2$, where $e_{i}^{(1)}$ and $e_{i}^{(2)}$ denote the error of one-step extrapolation by using the approximate solutions corresponding to $h=\frac{2 \pi}{10}$ and $h=\frac{2 \pi}{20}$ and to $h=\frac{2 \pi}{20}$ and $h=\frac{2 \pi}{40}$, respectively. The rate of convergence guaranteed by Theorem 2.4 is of order 5 .

TABLE 2. Errors of extrapolated solutions

\begin{tabular}{|c|c|c|c|}
\hline$t_{i}$ & $e_{i}^{(1)}$ & $e_{i}^{(2)}$ & rate \\
\hline 0.628319 & $-0.240441 \mathrm{D}-04$ & $-0.132255 \mathrm{D}-06$ & 7.51 \\
1.256637 & $-0.563067 \mathrm{D}-04$ & $-0.152681 \mathrm{D}-06$ & 8.53 \\
1.884956 & $-0.560020 \mathrm{D}-04$ & $-0.469600 \mathrm{D}-06$ & 6.90 \\
2.513274 & $-0.239315 \mathrm{D}-04$ & $-0.202012 \mathrm{D}-06$ & 6.89 \\
3.141593 & $-0.165464 \mathrm{D}-04$ & $-0.830877 \mathrm{D}-07$ & 7.64 \\
3.769911 & $-0.229695 \mathrm{D}-04$ & $0.730024 \mathrm{D}-06$ & 4.98 \\
4.398230 & $-0.571699 \mathrm{D}-04$ & $0.429444 \mathrm{D}-06$ & 7.06 \\
5.026548 & $-0.565343 \mathrm{D}-04$ & $0.136893 \mathrm{D}-06$ & 8.69 \\
5.654867 & $-0.243885 \mathrm{D}-04$ & $-0.488917 \mathrm{D}-07$ & 8.96 \\
6.283185 & $-0.167515 \mathrm{D}-04$ & $-0.368345 \mathrm{D}-07$ & 8.83 \\
\hline
\end{tabular}

Comparing Tables 1 and 2, we see that the error $e_{i}^{(1)}$ of one-step extrapolation is much smaller than the error $e_{i}$ of the Nyström solution with $h=2 \pi / 20$, and the error $e_{i}^{(2)}$ of one-step extrapolation is much smaller than the error $e_{i}$ of the Nyström solution with $h=2 \pi / 40$. Both Table 1 and Table 2 show that the convergence rates agree with theoretical estimates shown in Theorem 2.4. This example illustrates that the extrapolation process accelerates the order of convergence.

Example 2. In Example 1, if we choose $u(x, y)=e^{x} \cos y$, and $g=\frac{\partial u(P)}{\partial n_{P}}+u$ is calculated accordingly; then we have the following numerical results. Here we use the same notation as in the previous example. 
TABLE 3. Errors of the Nyström solutions

\begin{tabular}{|c|c|c|c|c|c|}
\hline$t_{i}$ & $e_{i}$ with $h=\frac{2 \pi}{10}$ & $e_{i}$ with $h=\frac{2 \pi}{20}$ & rate & $e_{i}$ with $h=\frac{2 \pi}{40}$ & rate \\
\hline 0.628319 & $-0.265869 \mathrm{D}-01$ & $-0.304603 \mathrm{D}-02$ & 3.13 & $-0.384348 \mathrm{D}-03$ & 2.99 \\
1.256637 & $-0.430752 \mathrm{D}-02$ & $-0.612696 \mathrm{D}-03$ & 2.81 & $-0.762916 \mathrm{D}-04$ & 3.01 \\
1.884956 & $-0.181330 \mathrm{D}-02$ & $-0.180546 \mathrm{D}-03$ & 3.33 & $-0.222135 \mathrm{D}-04$ & 3.02 \\
2.513274 & $-0.645594 \mathrm{D}-03$ & $-0.856597 \mathrm{D}-04$ & 2.91 & $-0.108117 \mathrm{D}-04$ & 2.99 \\
3.141593 & $0.316669 \mathrm{D}-02$ & $0.410404 \mathrm{D}-03$ & 2.95 & $0.505071 \mathrm{D}-04$ & 3.02 \\
3.769911 & $-0.645601 \mathrm{D}-03$ & $-0.865535 \mathrm{D}-04$ & 2.90 & $-0.107504 \mathrm{D}-04$ & 3.01 \\
4.398230 & $-0.181310 \mathrm{D}-02$ & $-0.181187 \mathrm{D}-03$ & 3.32 & $-0.239680 \mathrm{D}-04$ & 2.92 \\
5.026548 & $-0.430762 \mathrm{D}-02$ & $-0.612874 \mathrm{D}-03$ & 2.81 & $-0.761611 \mathrm{D}-04$ & 3.01 \\
5.654867 & $-0.265870 \mathrm{D}-01$ & $-0.304600 \mathrm{D}-02$ & 3.13 & $-0.384166 \mathrm{D}-03$ & 2.99 \\
6.283185 & $0.502972 \mathrm{D}-01$ & $0.590768 \mathrm{D}-02$ & 3.09 & $0.743281 \mathrm{D}-03$ & 2.99 \\
\hline
\end{tabular}

TABLE 4. Errors of extrapolated solutions

\begin{tabular}{|c|c|c|c|}
\hline$t_{i}$ & $e_{i}^{(1)}$ & $e_{i}^{(2)}$ & rate \\
\hline 0.628319 & $0.316946 \mathrm{D}-03$ & $-0.410773 \mathrm{D}-05$ & 6.27 \\
1.256637 & $-0.848634 \mathrm{D}-04$ & $0.337563 \mathrm{D}-06$ & 7.97 \\
1.884956 & $0.527035 \mathrm{D}-04$ & $0.405493 \mathrm{D}-06$ & 7.02 \\
2.513274 & $-0.566912 \mathrm{D}-05$ & $-0.119119 \mathrm{D}-06$ & 5.57 \\
3.141593 & $0.166489 \mathrm{D}-04$ & $-0.906675 \mathrm{D}-06$ & 4.20 \\
3.769911 & $-0.668961 \mathrm{D}-05$ & $0.786476 \mathrm{D}-07$ & 6.41 \\
4.398230 & $0.519436 \mathrm{D}-04$ & $-0.150824 \mathrm{D}-05$ & 5.11 \\
5.026548 & $-0.850536 \mathrm{D}-04$ & $0.512205 \mathrm{D}-06$ & 7.38 \\
5.654867 & $0.317002 \mathrm{D}-03$ & $-0.390363 \mathrm{D}-05$ & 6.34 \\
6.283185 & $-0.433684 \mathrm{D}-03$ & $0.551057 \mathrm{D}-05$ & 6.30 \\
\hline
\end{tabular}

\section{REFERENCES}

1. P. M. Anselone, Collectively Compact Operator Approximation Theory and Applications to Integral Equations, Prentice-Hall, Englewood Cliffs, N.J., 1971. MR 56:1753

2. K. E. Atkinson, The numerical solution of Fredholm integral equations of the second kind with singular kernels, Numer. Math. 19 (1972), 248-259. MR 46:6632

3. K. E. Atkinson, A Survey of Numerical Methods for the Solution of Fredholm Integral Equations of the Second Kind, SIAM, 1976. MR 58:3577

4. K. E. Atkinson, A survey of boundary integral equation methods for the numerical solution of Laplace's equation in three dimensions, in Numerical Solutions of Integral Equations, (Edited by Michael A. Golberg), Plenum, 1990. MR 91j:65169

5. K. E. Atkinson and G. Chandler, Boundary integral equation methods for solving Laplace's equation with nonlinear boundary conditions: the smooth boundary case, Math. Comp. 55 (1990), 451-472. MR 91d:65181

6. P. J. Davis and P. Rabinowitz, Methods of Numerical Integration, 2nd Edition, Academic Press, San Diego, 1984. MR 86d:65004

7. W. F. Ford and A. Sidi, An algorithm for a generalization of the Richardson extrapolation process, SIAM J. Numer. Anal. 24 (1987), 1212-1232. MR 89a:65006

8. L. Navot, An extension of the Euler-Maclaurin summation formula to functions with a branch singularity, J. Math. and Phys. 40 (1961), 271-276. MR 25:4290

9. L. Navot, A further extension of the Euler-Maclaurin summation formula, J. Math. and Phys. 41 (1962), 155-163.

10. K. Ruotsalainen and W. Wendland, On the boundary element method for some nonlinear boundary value problems, Numer. Math. 53 (1988), 299-314. MR 89h:65189 
11. C. Schneider, Regularity of the solution to a class of weakly singular Fredholm integral equations of the second kind, Int. Eqs. Op. Thy. 2 (1979), 62-68. MR 80f:45002

12. A. Sidi, Comparison of some numerical quadrature formulas for weakly singular periodic Fredholm integral equations, Computing 43 (1989), 159-170. MR 91c:65086

13. A. Sidi and M. Israeli, Quadrature methods for periodic singular and weakly singular Fredholm integral equations, J. Scientific Computing 3 (1988), 201-231. MR 90e:65194

14. I. Sloan, Private communication, 1993.

Department of Mathematics, North Dakota State University, Fargo, North Dakota 58105

E-mail address, Y. Xu: xu@plains.Nodak.edu

E-mail address, Y. Zhao: yunhe@plains.Nodak.edu 